ROCKY MOUNTAIN

JOURNAL OF MATHEMATICS

Volume 45, Number 4, 2015

\title{
ON 2-SG-SEMISIMPLE RINGS
}

\author{
DRISS BENNIS, KUI HU AND FANGGUI WANG
}

\begin{abstract}
In this paper, we investigate 2-SG-semisimple rings which are a particular kind of quasi-Frobenius rings over which all modules are periodic of period 2. Namely, we show that local 2-SG-semisimple rings are the same as the known Artinian valuation rings. Also, a relation between Dedekind domains and 2-SG-semisimple rings is established.
\end{abstract}

1. Introduction. Throughout this paper, all rings are commutative with identity element and all modules are unital. It is convenient to use $m$-local or (simply) local to refer to not necessarily Noetherian rings with a unique maximal ideal $m$. We assume that the reader is familiar with the Gorenstein homological algebra (some references are $[9,10,12])$.

For a ring $R$ and a positive integer $n \geq 1$, an $R$-module $M$ is said to be $n$-strongly Gorenstein projective ( $n$-SG-projective for short), if there exists an exact sequence of $R$-modules

$$
0 \longrightarrow M \longrightarrow P_{n} \longrightarrow \cdots \longrightarrow P_{1} \longrightarrow M \longrightarrow 0,
$$

where each $P_{i}$ is projective, such that $\operatorname{Hom}_{R}(-, Q)$ leaves the sequence exact whenever $Q$ is a projective $R$-module (see [6]). The 1-SGprojective module is simply called strongly Gorenstein projective (SGprojective for short) (see [5]). An extension of these kinds of modules was given in [3]. Namely, we have, for integers $n \geq 1$ and $m \geq 0$, a module $M$ is called $(n, m)-S G$-projective if there exists an exact sequence of modules,

$$
0 \longrightarrow M \longrightarrow Q_{n} \longrightarrow \cdots \longrightarrow Q_{1} \longrightarrow M \longrightarrow 0,
$$

2010 AMS Mathematics subject classification. Primary 13C13, 13F05, 13F10, 13H10, 13F30, Secondary 03E75.

Keywords and phrases. 2-SG-projective, 2-SG-semisimple rings, quasi-Frobenius rings, factors of Dedekind domains, Artinian valuation rings.

Received by the editors on January 2, 2013, and in revised form on July 23, 2013.

DOI:10.1216/RMJ-2015-45-4-1093

Copyright (C)2015 Rocky Mountain Mathematics Consortium 
where $\operatorname{pd}\left(Q_{i}\right) \leq m$ for $1 \leq i \leq n$, such that $\operatorname{Ext}^{i}(M, Q)=0$ for any $i>m$ and for any projective module $Q$. A general study of rings over which every module is $(n, m)$-SG-projective was done in [4], and such rings are called $(n, m)$-SG. Thus, as in classical homological dimension, the $(n, m)$-SG rings with small integers $n$ and $m$ would be of interest. Let us call by $n$-SG-semisimple, for an integer $n \geq 1$, the $(n, 0)$-SG rings. From [4, Corollary 2.8], $n$-SG-semisimple rings are a particular kind of quasi-Frobenius rings. In [8], it was proved that a local ring is 1-SG-semisimple if and only if it contains a unique non-trivial ideal.

The aim of this paper is to study 2-SG-semisimple rings. We prove that 2-SG-semisimple is the same as the well-known Artinian serial rings (see Corollary 2.7). Recall that a ring is called serial if it is a finite direct product of valuation rings, where a ring (not necessarily a domain) is called valuation if the lattice of all its ideals is linearly ordered under inclusions (see, for example, [11, pages 10 and 11]). Namely, we prove that a local ring is 2-SG-semisimple if and only if it is an Artinian valuation ring (see Theorem 2.6). Also, a relation between Dedekind domains and 2-SG-semisimple rings is established in Proposition 2.9.

Before starting, we need to recall some useful results about quasiFrobenius rings (for more details about these kinds of rings, see, for example, [14]). The quasi-Frobenius rings have several characterizations, and here, we only need the following ones:

Theorem 1.1 ([14], Theorems 1.50, 7.55 and 7.56). For a ring $R$, the following are equivalent:

(i) $R$ is quasi-Frobenius;

(ii) $R$ is Artinian and self-injective;

(iii) every projective $R$-module is injective;

(iv) every injective $R$-module is projective;

(v) $R$ is Noetherian and, for every ideal I, Ann $(\operatorname{Ann}(I))=I$, where Ann $(I)$ denotes the annihilator of $I$.

For the local case, we have the following result:

Theorem 1.2 ([13], Theorems 221). Let $R$ be an m-local and zerodimensional Noetherian ring. The following are equivalent: 
(i) $R$ is quasi-Frobenius;

(ii) $\operatorname{Ann}(m)$ is a principal ideal.

We have the following structural characterization of quasi-Frobenius rings.

Proposition 1.3. A ring $R$ is quasi-Frobenius if and only if $R=$ $R_{1} \times \cdots \times R_{n}$, where each $R_{i}$ is a local quasi-Frobenius ring.

2. Main results. We aim to give an equivalent characterization of 2-SG-semisimple rings. The following leads us to restrict the study to the case of local rings.

Lemma 2.1 ([4], Proposition 2.13). $A$ ring $R$ is 2-SG-semisimple if and only if $R=R_{1} \times \cdots \times R_{n}$, where each $R_{i}$ is a local 2-SG-semisimple ring.

Before giving the main result, we need the following lemmas.

The following result is a characterization of Artinian valuation local rings.

Lemma 2.2 ([2], Proposition 8.8). Let $R$ be an Artinian m-local ring. Then the following assertions are equivalent:

(i) every ideal is principal;

(ii) the maximal ideal $m$ is principal;

(iii) $R$ is a valuation ring.

In this case every ideal $I$ of $R$ is of the form $a^{n} R$ where a generates $m$.

The two results below investigate the 2-SG-projective modules over local quasi-Frobenius rings.

Lemma 2.3. Let $R$ be a local quasi-Frobenius ring and $M$ a finitely generated $R$-module. If $M$ is 2-SG-projective, then there is an exact sequence $0 \rightarrow M \rightarrow F_{2} \rightarrow F_{1} \rightarrow M \rightarrow 0$ where $F_{1}$ and $F_{2}$ are free and 
finitely generated $R$-modules. Furthermore, if $M$ is an ideal of $R$, then the exact sequence can be of the form:

$$
0 \longrightarrow M \longrightarrow R \longrightarrow R^{n} \longrightarrow M \longrightarrow 0,
$$

where $n$ is a positive integer.

Proof. Let $M$ be a finitely generated 2-SG-projective $R$-module. Then, by [18, Theorem 3.14], there exists an exact sequence of $R$ modules

$$
0 \longrightarrow M \longrightarrow F_{2} \longrightarrow F_{1} \longrightarrow M \longrightarrow 0
$$

with $F_{1}$ and $F_{2}$ are finitely generated projective $R$-modules. Notice that $R$ is local, so $F_{1}$ and $F_{2}$ are finitely generated free and the first assertion follows.

Now, suppose that $M$ is an ideal of $R$. Decomposing the exact sequence $0 \rightarrow M \rightarrow F_{2} \rightarrow F_{1} \rightarrow M \rightarrow 0$ to get the short exact sequences: $0 \rightarrow M \rightarrow F_{2} \rightarrow K \rightarrow 0$ and $0 \rightarrow K \rightarrow F_{1} \rightarrow M \rightarrow 0$. Since $R$ is quasi-Frobenius, $F_{1}$ and $R$ are injective $R$-modules. Then, we can apply the dual of the horseshoe lemma [15, Note after Lemma 6.20] to the short exact sequences above with the canonical one, $0 \rightarrow M \rightarrow R \rightarrow R / M \rightarrow 0$, to get the following commutative diagram with exact columns and rows:

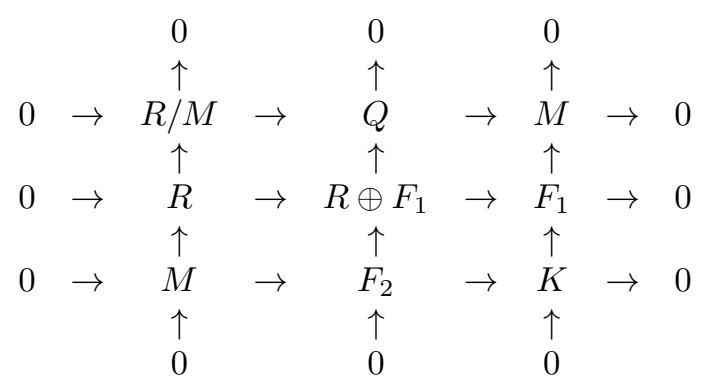

From the top horizontal sequence, $Q$ is a Gorenstein projective and finitely generated $R$-module. Then, using the middle vertical sequence, $Q$ has finite projective dimension. This shows, using [12, Proposition 2.27 ], that $Q$ is projective and then free (since $R$ is local). Then, there is a positive integer $n$ such that $Q \cong R^{n}$. Finally, combining the top horizontal sequence with the left vertical one to get the desired sequence. 
Corollary 2.4. Let $R$ be a local quasi-Frobenius ring, and let a be a zero-divisor element of $R$. If the principal ideal a $R$ is 2-SG-projective, then $\operatorname{Ann}(a)$ is also principal and there are exact sequences of the form:

$$
\begin{aligned}
& 0 \longrightarrow a R \longrightarrow R \longrightarrow R \longrightarrow a R \longrightarrow 0 \\
& 0 \longrightarrow \operatorname{Ann}(a) \longrightarrow R \longrightarrow R \longrightarrow \operatorname{Ann}(a) \longrightarrow 0 \\
& 0 \longrightarrow R / a R \longrightarrow R \longrightarrow R \longrightarrow a R \longrightarrow 0
\end{aligned}
$$

Proof. By Lemma 2.3, we have an exact sequence of the form:

$$
0 \longrightarrow R / a R \longrightarrow R^{n} \longrightarrow a R \longrightarrow 0
$$

where $n$ is a positive integer. By the Schanuel lemma [15, Theorem 9.4 (i)], the above exact sequence with the following canonical one:

$$
0 \longrightarrow \operatorname{Ann}(a R) \longrightarrow R \longrightarrow a R \longrightarrow 0
$$

implies that $\operatorname{Ann}(a) \oplus R^{n} \cong R / a R \oplus R$. This shows that Ann $(a)$ must be principal and $n=1$ which help to construct the desired sequences.

The structure of modules over Artinian serial rings is given by the following well-known result.

Lemma 2.5 ([11], Theorems 5.6). Let $R$ be an Artinian serial ring. Then every $R$-module is a direct sum of cyclic modules.

Now we are in position to give the main result.

Theorem 2.6. An m-local ring $R$ is 2-SG-semisimple if and only if it is an Artinian valuation ring.

Proof. If $R$ is 2-SG-semisimple, then it is quasi-Frobenius (by [4, Corollary 2.8]). Then, by Theorem 1.2, Ann $(m)$ is principal. This shows, using Corollary 2.4 and Theorem 1.1, that $m=\operatorname{Ann}(\operatorname{Ann}(m))$ is principal. Therefore, $R$ is a valuation ring (by Lemma 2.2). Conversely, assume that $R$ is an Artinian valuation ring. Obviously, $R$ is quasi-Frobenius with only principal ideals. Then, for every zero-divisor element $a$ of $R$, we have the exact sequences $0 \rightarrow \operatorname{Ann}(a) \rightarrow R \rightarrow$ 
$a R \rightarrow 0$ and $0 \rightarrow a R=\operatorname{Ann}(\operatorname{Ann}(a)) \rightarrow R \rightarrow \operatorname{Ann}(a) \rightarrow 0$. Combining these sequences, we deduce that $a R$ is 2 -SG-projective. Then, from Corollary 2.4, the cyclic module $R / a R$ is also 2-SG-projective and so are all cyclic modules including the free ones. Therefore, Lemma 2.5 with [3, Proposition 2.3] show that every module is 2-SG-projective and therefore $R$ is 2 -SG-semisimple.

From Lemma 2.1, the structure of 2-SG-semisimple rings is immediately deduced as follows.

Corollary 2.7. A ring $R$ is 2-SG-semisimple if and only if it is an Artinian serial ring.

To construct examples of 2-SG-semisimple rings, one can use the well-known result that nontrivial factor rings of Dedekind domains are principal Artinian serial rings, which means that nontrivial factor rings of Dedekind domains are 2-SG-semisimple (see, for example, [17, Corollary, page 278]). The following result (Proposition 2.9) shows that the Dedekind domains is closely related to the 2-SG-semisimple rings in the sense that the converse of the well-known result above holds true. To prove this result, we use the following lemma.

Lemma 2.8. Let $R$ be a domain and $P$ a maximal ideal of $R$ which is finitely generated. Then $P$ is invertible if and only if $P_{P}\left(\right.$ i.e., $\left.P R_{P}\right)$ is a principal ideal of $R_{P}$.

Proof. By [16, Theorem 8.4.2], $P$ is invertible if and only if $P_{\mathfrak{m}}$ is principal for any maximal ideal $\mathfrak{m}$ of $R$. Since $P$ is maximal, $P_{\mathfrak{m}}=R_{\mathfrak{m}}$ for any maximal ideal $\mathfrak{m}$ other than $P$.

Proposition 2.9. A domain $R$ is Dedekind if and only if every nontrivial factor ring of $R$ is 2-SG-semisimple.

Proof. If every nontrivial factor ring of $R$ is 2-SG-semisimple, then, by [13, Theorem 90], $R$ must be one-dimensional and Noetherian. So, by [1, Theorem 3], $R$ must be a Dedekind domain. We give a direct proof here. Let $P$ be a maximal ideal of $R$, and let $a$ be an element in $P$ which is not in $P^{2}$. Since $R / P^{2}$ is a QF-ring, by Theorem 1.1, $(\bar{a})=\operatorname{Ann}(\operatorname{Ann}(\bar{a}))$. Since $\left(P / P^{2}\right)^{2}=0$, it can be seen 
that Ann $(\operatorname{Ann}(\bar{a}))=P / P^{2}$. Therefore $(\bar{a})=P / P^{2}$. So $R a+P^{2}=P$ and by the Nakayama lemma, $P_{P}=(a)_{P}$. Thus, by Lemma $2.8, P$ is invertible, and this means that $R$ is a Dedekind domain.

For the "only if" part, let $I$ be a proper ideal of a Dedekind domain $R$. Then $I=P_{1}^{t_{1}} P_{2}^{t_{2}} \cdots P_{n}^{t_{n}}$ for some prime ideals $P_{1}, P_{2}, \cdots, P_{n}$ and some integers $t_{1}, t_{2}, \cdots, t_{n}$. By the Chinese remainder theorem, $R / I \cong R / P_{1}^{t_{1}} \oplus R / P_{2}^{t_{2}} \bigoplus \cdots \oplus R / P_{n}^{t_{n}}$. In order to show that $R / I$ is 2-SG-semisimple, we only need to prove that $R / P_{i}^{t_{i}}$ is such a ring. When $t_{i}=1$, the field $R / P_{i}$ is certainly $2-\mathrm{SG}$-semisimple. Therefore, we can assume that $t_{i}>1$. Since $R / P_{i}^{t_{i}}$ is an Artinian local ring, by Lemma 2.2 and Theorem 2.6, it suffices to prove that the maximal ideal $P_{i} / P_{i}^{t_{i}}$ is principal. By [16, Corollary 9.8.7], we can choose an element $b \in P_{i}^{t_{i}}$ and an element $c \in P_{i}$ such that $P_{i}=(b, c)$. Therefore, $P_{i} / P_{i}^{t_{i}}=\left(c+P_{i}^{t_{i}}\right)$ is principal.

Acknowledgments. The authors would like to thank the referee for careful reading and helpful comments.

\section{REFERENCES}

1. Z. Abraham, Restricted Gorenstein rings, Illinois J. Math. 13 (1969), 796803.

2. M. Atiyah and I.G. Macdonald, Introduction to commutative algebra, Addison Wesley, Reading, 1969.

3. D. Bennis, $(n, m)$-Strongly Gorenstein projective modules, Int. Electr. J. Alg.6 (2009), 119-133.

4. , $(n, m)-S G$ rings, AJSE-Mathematics 35 (2010), 169-178.

5. D. Bennis and N. Mahdou, Strongly Gorenstein projective, injective, and flat modules, J. Pure Appl. Alg. 210 (2007), 437-445.

6. , A generalization of strongly Gorenstein projective modules, J. Alg. Appl. 8 (2009), 219-227.

7. $461-465$. , Global Gorenstein dimensions, Proc. Amer. Math. Soc. 138 (2010),

8. D. Bennis, N. Mahdou and K. Ouarghi, Rings over which all modules are strongly Gorenstein projective, Rocky Mountain J. Math. 40 (2010), 749-759.

9. L.W. Christensen, Gorenstein dimensions, Lect. Notes Math. 1747, SpringerVerlag, Berlin, 2000.

10. E.E. Enochs and O.M.G. Jenda, Relative homological algebra, de Gruyter Expo. Math. 30, Walter de Gruyter \& Co., Berlin, 2000.

11. A. Facchini, Module theory: Endomorphism rings and direct sum decompositions in some classes of modules, Birkhaüser, Basel, 1998. 
12. H. Holm, Gorenstein homological dimensions, J. Pure Appl. Alg. 189 (2004), $167-193$.

13. I. Kaplansky, Commutative rings, Allyn and Bacon, Boston, 1970.

14. W.K. Nicholson and M.F. Youssif, Quasi-Frobenius rings, Cambridge University Press, Cambridge, 2003.

15. J.J. Rotman, An introduction to homological algebra, Academic Press, New York, 1979.

16. F. Wang, Commutative rings and star operation theory, Science Press, Beijing, 2006 (in Chinese).

17. O. Zariski and P. Samuel, Commutative algebra, Vol. 1, Springer New York, 1975.

18. G.Q. Zhao and Z.Y. Huang, $n$-strongly Gorenstein projective, injective and flat modules, Comm. Alg. 39 (2011), 3044-3062.

Department of Mathematics, Faculty of Sciences, B.P. 1014, Mohammed V University, RABAT, MOROCCO

Email address: d.bennis@fsr.ac.ma, driss_bennis@hotmail.com

College of Science, Southwest University of Science and Technology, Mianyang, 621010, P.R. China

Email address: hukui200418@163.com

College of Mathematics and Software Science, Sichuan Normal UniverSity, Chengdu, 610068, P.R. China

Email address: wangfg2004@163.com 